\title{
介观结构氮掺杂碳纳米笼负载铂-钉合金催化剂的优异甲醇电氧化性能
}

\author{
黎聑勤 \\ 张志琦 \\ 藏鹏远 \\ 马延文吴强 \\ 杨立军 \\ 陈强王喜章* 胡征
}

(江苏省纳米技术重点实验室 介观化学教育部重点实验室 南京大学化学化工学院 南京 210023; 南京大学(苏州)

高新技术研究院 苏州 215123)

\begin{abstract}
摘要 利用氮掺杂碳纳米笼( $\mathrm{hNCNC}$ )的高比表面积及掺杂氮原子的针定作用, 方便地将约 $3 \mathrm{~nm}$ 的 Pt-Ru 合金纳米粒子 均匀地负载在 $\mathrm{hNCNC}$ 表面，制得了 $\mathrm{Pt}$ 和 $\mathrm{Ru}$ 比例可调的 Pt-Ru/hNCNC 双金属合金催化剂. 这些催化剂展现出优异的 甲醇催化氧化活性和稳定性，且具有良好的抗 $\mathrm{CO}$ 中毒能力，显著优于 $\mathrm{Pt} / \mathrm{hNCNC}$ 和商业 $\mathrm{PtRu} / \mathrm{C}$ 等对照组催化剂. 其优 异的电化学性能可归因于以下因素的协同作用: (1) Pt-Ru 合金的双功能机制增强了催化剂的 CO 氧化脱附能力从而使 活性位重新暴露, (2) hNCNC 的氮掺杂及高比表面积有利于获得粒径小且均匀的合金纳米粒子, (3) hNCNC 的多尺度分 级孔结构有利于甲醇等参与反应物质的传输.

关键词 直接甲醇燃料电池; 电催化剂; 介观结构; 氮掺杂碳纳米笼; 铂钉合金; 甲醇氧化
\end{abstract}

\section{Alloyed Pt-Ru Nanoparticles Immobilized on Mesostructured Nitrogen-Doped Carbon Nanocages for Efficient Methanol Electrooxidation}

\author{
Li, Danqin Zhang, Zhiqi Zang, Pengyuan Ma, Yanwen Wu, Qiang Yang, Lijun \\ Chen, Qiang Wang, Xizhang* Hu, Zheng \\ (Jiangsu Provincial Lab for Nanotechnology, Key Laboratory of Mesoscopic Chemistry of MOE, School of Chemistry and \\ Chemical Engineering, Nanjing University, Nanjing 210023, China. High-Tech Research Institute of \\ Nanjing University (Suzhou), Suzhou, Jiangsu 215123)
}

\begin{abstract}
Direct methanol fuel cells (DMFC) have attracted extensive attention as ideal candidates for automotive and portable applications owing to the fascinating advantages such as high conversion efficiency, environmental friendliness, safety, wide sources of methanol, and simple cell structure. Electrocatalysts are one of crucial factors limiting the performance of DMFC. Nowadays, precious Pt-based catalyst, in spite of costliness and scarcity, is the most popular catalyst for methanol oxidation reaction (MOR) at anode due to the much better performances than those of the non-Pt catalysts. But there exists some shortcomings such as poor CO-tolerance and durability. Pt alloying with other metals, e.g. Ru, is an effective strategy to improve the catalytic performance. In addition, the support with a large specific surface area (SSA), high conductivity and suitable porous structure, such as $\mathrm{sp}^{2}$ carbon, could lead to high dispersion, high utilization and stability of Pt-based nanoparticles, also favorable for MOR. Recently, by in situ MgO template method, we reported the unique 3D hierarchical carbon-based nanocages featured with ultrahigh SSA, micro-meso-macro-pore coexistence, good conductivity and easy doping, which exhibited excellent electrochemical performances. Herein, taking the advantages of nitrogen-dopant anchoring function and unique mesostructures of hierarchical N-doped carbon nanocages (hNCNC), we report the Pt-Ru electrocatalysts immobilized on $\mathrm{hNCNC}(\mathrm{Pt}-\mathrm{Ru} / \mathrm{hNCNC}$ ) prepared via modified microwave-assisted ethylene glycol (EG) reduction method. The so-constructed $\mathrm{Pt}-\mathrm{Ru} / \mathrm{hNCNC}$ catalysts with $c a .30 \mathrm{wt} \%$ loading and tunable atomic ratio of $\mathrm{Pt}$ to Ru have a highly homogeneous dispersion of metal nanoparticles with the average size of $c a .3 \mathrm{~nm}$. The alloying Pt-Ru/hNCNC catalysts demonstrate good CO-tolerance, high MOR activity and durability, superior to those of the counterparts of Pt/hNCNC and commercial PtRu/C. The good electrochemical performance can be ascribed to the synergistic effects of the bifunctional effect due to introduction of Ru, small size and high dispersion of metal nanoparticles induced by the large SSA and nitrogen participation of hNCNC, and multi-scaled hierarchical pore structures beneficial to the mass transportation. These results proposed a potential strategy to develop the high-performance Pt-based MOR catalysts based on the novel mesostructured hNCNC.
\end{abstract}

Keywords direct methanol fuel cell; electrocatalyst; mesostructured; nitrogen-doped carbon nanocages; Pt-Ru alloy nano-

* E-mail: wangxzh@nju.edu.cn; Tel.: 025-89683696; Fax: 025-89683696

Received April 20, 2016; published May 25, 2016.

Project supported by the National Basic Research Program of China (973 Program, No. 2013CB932902), National Natural Science Foundation of China (Nos 21473089, 51232003, 21373108, 51571110, 21573107), Suzhou Science and Technology Project (ZXG2013025) and Changzhou Technology Support Program (CE20130032). This work was also supported by a Project Funded by the Priority Academic Program Development of Jiangsu Higher Education Institutions.

项目受国家重大科学研究计划纳米专项(No. 2013CB932902)、国家自然科学基金(Nos. 21473089, 51232003, 21373108, 51571110, 21573107)、苏州市 科技计划(ZXG2013025)、常州市科技计划(CE20130032)和江苏高校优势学科建设工程资助. 


\section{1 引言}

直接甲醇燃料电池(DMFC) 具有能量转换效率高、 环境友好、低温启动速度快、燃料甲醇易输运且来源广 泛、电池结构简单等优点, 在便携式电源等方面有广阔 应用前景 ${ }^{[1,2]}$. DMFC 的阳极发生甲醇氧化反应(MOR), 通常采用 $\mathrm{Pt} / \mathrm{C}$ 催化剂. $\mathrm{Pt} / \mathrm{C}$ 催化剂的活性高, 但存在稳 定性差、抗 $\mathrm{CO}$ 中毒能力低等不足, 因此, 开发催化活 性高、抗 $\mathrm{CO}$ 中毒能力强和稳定性好的催化剂尤显重要. 将 $\mathrm{Pt}$ 与其他金属 $\left(\mathrm{Ru}^{[3]} 、 \mathrm{Sn}^{[4]} 、 \mathrm{~W}^{[5]} 、 \mathrm{Cu}^{[6]} 、 \mathrm{Mo}^{[7]}\right.$ 等$)$ 合 金化构建双元或多元合金催化剂能够有效降低成本, 优 化电催化性能. 其中, Pt-Ru 催化剂因具有优异的甲醇氧 化催化活性和良好的抗 CO 中毒能力而备受关注. 金属 $\mathrm{Ru}$ 的引入能够显著增强 $\mathrm{Pt}$ 催化剂的 $\mathrm{CO}$ 氧化脱附能力, 使其活性位重新暴露, 这就是目前被广泛接受的 Pt-Ru 催化剂抗中毒性能的双功能机制 ${ }^{[8]}$. 调变载体的结构和 组成是提高 Pt 基催化剂 MOR 性能的另一条有效途径. 理想的载体应具有以下特征: 大比表面积、合适的金属载体相互作用和孔结构. 这些特征有利于金属纳米粒子 在载体表面的均匀分散、固载、减小粒径、增加电活性 表面积、参与反应物质的传输等, 进而促进催化剂的电 催化性能 ${ }^{[9 \sim 13]}$. 碳材料具有来源广泛、形态结构丰富、 导电性好、抗腐蚀能力强、比表面积和孔结构易于调控、 还易于掺杂等特点, 是构建高性能 MOR 催化剂的常用 载体, 包括碳基纳米管 ${ }^{[10,14,15]}$ 、多孔碳 ${ }^{[16 ~ 19]}$ 、碳气凝 胶 ${ }^{[20]}$ 、石墨烯 ${ }^{[21]}$ 和纳米金刚石 ${ }^{[22]}$ 等. 在石墨碳层中引入 杂原子能够有效引入针定位点, 促进催化活性物种的分 散, 调节金属-载体的相互作用, 从而调变电催化性 能 ${ }^{[9-11]}$. 我们的前期研究表明, 无需对氮掺杂碳纳米管 (NCNTs)进行任何预处理, 通过简便的乙二醇还原法就 可将 $\mathrm{Pt}$ 基纳米粒子(Pt、Pt-Co、Pt-Ru 等)均匀地负载在 NCNTs 表面. 这些催化剂展现出优异的 $\mathrm{MOR}^{[9,10]}$ 和氧 还原(ORR $)^{[23]}$ 电催化性能. 然而, NCNTs 的比表面积一 般仅约 $200 \mathrm{~m}^{2} \cdot \mathrm{g}^{-1}$, 在碳基载体中并不突出. 最近, 我 们课题组通过原位 $\mathrm{MgO}$ 模板法制备出了具有比表面积 高 $\left(>1000 \mathrm{~m}^{2} \cdot \mathrm{g}^{-1}\right)$ 、导电性好、微孔一介孔-大孔共存等 特征的分级结构碳基纳米笼, 在能源转化和存储等方面

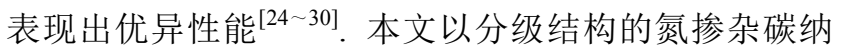
米笼 $(\mathrm{hNCNC})$ 为载体, 通过微波辅助乙二醇还原法将 $\mathrm{Pt}-\mathrm{Ru}$ 合金纳米粒子高度分散于该载体, 并调变了 $\mathrm{Pt}$ 、 $\mathrm{Ru}$ 的原子比. 这些催化剂展现出优异的 MOR 催化活性 和稳定性以及良好的抗 $\mathrm{CO}$ 中毒能力, 优于 $\mathrm{Pt} / \mathrm{hNCNC}$ 和商业 $\mathrm{PtRu} / \mathrm{C}(20 \mathrm{wt} \%)$ 等对照组催化剂.

\section{2 结果与讨论}

以 $\mathrm{hNCNC}$ 为载体, 通过微波辅助乙二醇还原法将
$\mathrm{Pt}-\mathrm{Ru}$ 合金纳米粒子分散在 $\mathrm{hNCNC}$ 载体上. 金属物种的 总投料量为 $30 \mathrm{wt} \%$, 通过改变投料比调变 $n(\mathrm{Pt}): n(\mathrm{Ru})$ 的物质的量比. 电感耦合等离子体原子发射光谱 (ICP-AES) 测试结果表明, Pt/hNCNC、 $\mathrm{Pt}_{2} \mathrm{Ru} / \mathrm{hNCNC}$ 、 $\mathrm{PtRu} / \mathrm{hNCNC} 、 \mathrm{PtRu} / \mathrm{hNCNC}$ 和 $\mathrm{Ru} / \mathrm{hNCNC}$ 中的金属总 负载量依次为 $29.2 、 29.5 、 28.9 、 29.1$ 和 $29.5 \mathrm{wt} \%$, 三 个合金催化剂的 $n(\mathrm{Pt}): n(\mathrm{Ru})$ 物质的量比依次为 2.0 : $0.9 、 1.0: 1.1$ 和 $1.0: 2.1$. 总负载量及 $n(\mathrm{Pt}): n(\mathrm{Ru})$ 物质 的量比均与投料比接近.

图 1 是 $\mathrm{hNCNC}$ 载体和 $\mathrm{Pt}-\mathrm{Ru} / \mathrm{hNCNC}$ 系列催化剂的 结构表征结果. 透射电子显微镜(TEM)及扫描电子显微 镜(SEM)照片显示, hNCNC 呈多孔球形介观结构, 由尺 寸约 $40 \mathrm{~nm}$ 的空心纳米笼相连成纳米片、进而组装而 成 ${ }^{[27,28]}$. 纳米笼壁由约 $6 \sim 8$ 层碳层构成, 石墨化程度良 好(图 1a). 氮气吸附-脱附等温线和孔分布曲线表明, $\mathrm{hNCNC}$ 中微孔、介孔、大孔共存, 比表面积为 1230 $\mathrm{m}^{2} \cdot \mathrm{g}^{-1}$, 其中微孔尺寸约为 $0.6 \mathrm{~nm}$, 主要来自穿过笼壁 的孔, 介孔尺寸约 $35 \mathrm{~nm}$, 主要来自笼内空腔和笼间堆 积, 尺寸大于 $50 \mathrm{~nm}$ 的大孔主要来自片层堆积孔和球状 颗粒堆积孔(图 1d) ${ }^{[24,25,27 ~ 30]}$. hNCNC 的高比表面积有利 于金属纳米粒子的高度分散, 多尺度分级孔结构有利于 甲醇等参与反应物质的传输, 高导电性有利于电子传 输, 这三者可协同促进电催化反应的进行. TEM 结果显 示 $\mathrm{Pt}$ 和 $\mathrm{PtRu}$ 纳米粒子的尺寸约为 $3 \mathrm{~nm}$, 均匀地分散在 $\mathrm{hNCNC}$ 表面(图 1b,1c). 高分辨 TEM(HRTEM)结果显 示, PtRu 纳米粒子的晶面间距为 $0.223 \mathrm{~nm}$, 比纯 $\mathrm{Pt}$ 的 (111)面间距 $(0.230 \mathrm{~nm}$ )略小(图 $1 \mathrm{~b}, 1 \mathrm{c})$, 这是因为 $\mathrm{Ru}$ 的 原子半径(1.34 $\AA$ ) 比 $\operatorname{Pt}(1.39 \AA)$ 小, 形成合金后使得晶面 间距缩小. X-射线衍射谱(XRD)显示，位于 $39.7^{\circ} 、 45.4^{\circ}$ 、 $67.4^{\circ}$ 和 $81.1^{\circ}$ 的衍射峰可归属为面心立方结构 $\mathrm{Pt}$ 的 (111)、(200)、(220)和(311)晶面(图 1e); 随合金中 $\mathrm{Ru}$ 含 量逐渐增加, 各衍射峰逐渐向高角度偏移, 没有出现纯 $\mathrm{Ru}$ 的衍射峰. XRD 和 HRTEM 结果证实了 $\mathrm{Pt}$ 和 $\mathrm{Ru}$ 的合 金化(图 1b,1c,1e). XRD 衍射谱 $26^{\circ}$ 附近较强的衍射峰 可归属为石墨的(002)晶面, 说明 $\mathrm{hNCNC}$ 具有良好的石 墨化程度.

图 2 是 Pt-Ru/hNCNC 系列催化剂的 $\mathrm{X}$ 射线光电子 能谱(XPS). Pt/hNCNC 的 Pt4f 精细谱可拟合为两组峰, 分别对应于 $\operatorname{Pt}(0)$ 和 $\operatorname{Pt}(\mathrm{II})$ 物种的 $4 \mathrm{f}_{7 / 2}$ 和 $4 \mathrm{f}_{5 / 2}$ (图 2a) ${ }^{[31]}$. $\mathrm{Ru} / \mathrm{hNCNC}$ 的 Ru3p 精细谱可归属为 $\mathrm{Ru}(0)$ 和 $\mathrm{Ru}(\mathrm{IV})$ 物 种(图 2b) ${ }^{[31]}$. 这两个样品中金属态 $\operatorname{Pt}(0)$ 和 $\mathrm{Ru}(0)$ 占主导, 与 HRTEM 和 XRD 结果相一致(图 $1 b, 1 c, 1 e$ ), 表明在微 波辅助乙二醇反应条件下, $\mathrm{Pt}$ 和 $\mathrm{Ru}$ 盐能被有效地还原 成零价的金属. 对比该系列样品的 $\mathrm{Pt} 4 \mathrm{f}$ 和 $\mathrm{Ru} 3 \mathrm{p}_{3 / 2}$ 精细 谱可知，随着 Pt-Ru 合金中 $\mathrm{Ru}$ 含量的增加, $\mathrm{Pt} 4 \mathrm{f}$ 峰强度 下降, Ru 3p 峰强度升高; Pt $4 \mathrm{f}$ 的结合能逐渐向高结合能 

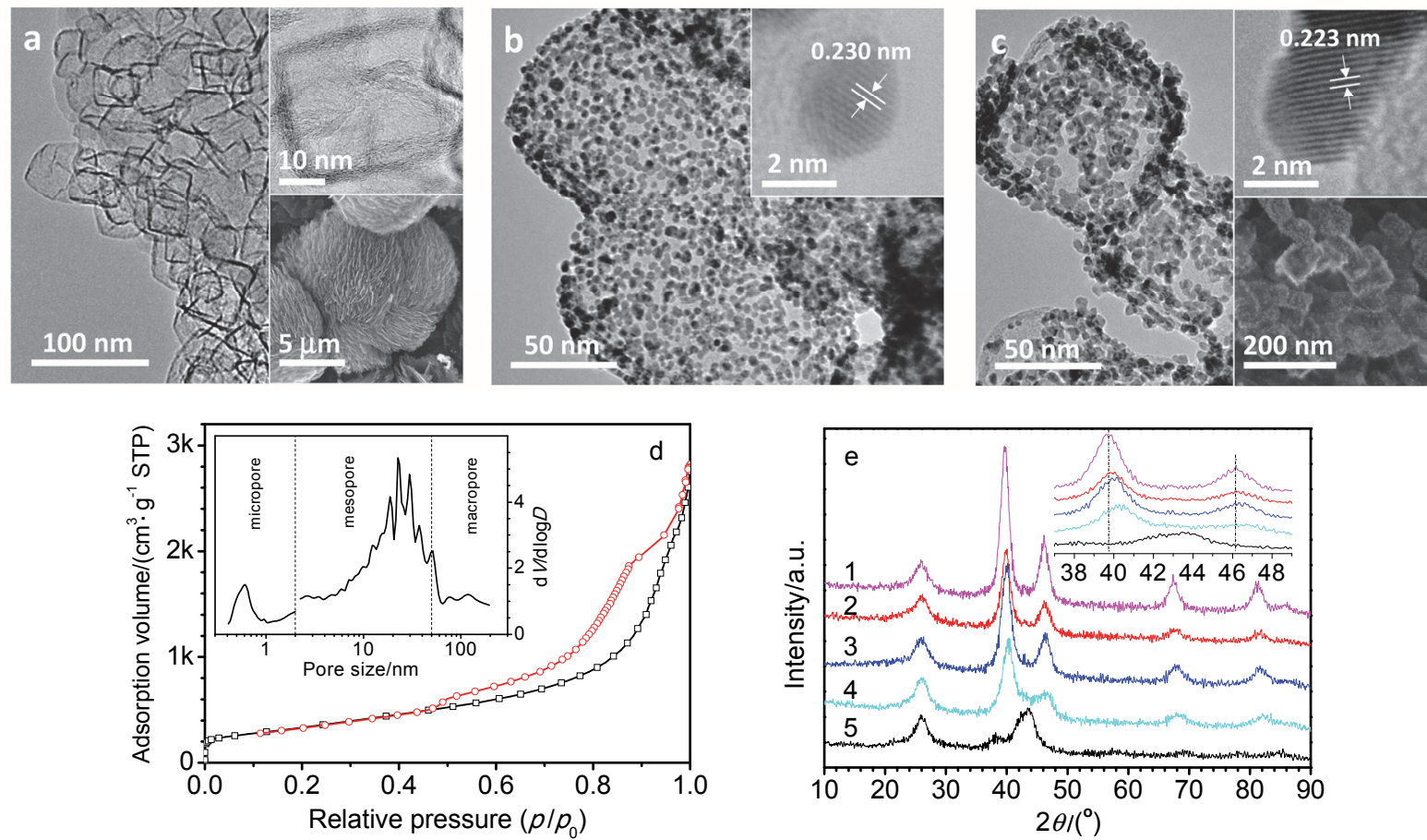

图 $1 \mathrm{hNCNC}$ 载体及 Pt-Ru/hNCNC 催化剂的结构表征. (a c) hNCNC、Pt/hNCNC 和 PtRu/hNCNC 的 TEM 照片. 右上角插图为 HRTEM, (a, c) 的右下角插图为 SEM. (d) hNCNC 的氮气吸附-脱附等温线和孔分布曲线. (e) Pt-Ru/hNCNC 系列样品的 XRD 衍射谱. $1 \sim 5$ 对应于 Pt/hNCNC、 $\mathrm{Pt}_{2} \mathrm{Ru} / \mathrm{hNCNC} 、 \mathrm{PtRu} / \mathrm{hNCNC} 、 \mathrm{PtRu} / \mathrm{hNCNC}$ 和 $\mathrm{Ru} / \mathrm{hNCNC}$ (下同)

Figure 1 Structure characterizations of Pt-Ru/hNCNC catalysts and support. $(\mathrm{a} \sim \mathrm{c}) \mathrm{TEM}$ images of $\mathrm{hNCNC}, \mathrm{Pt} / \mathrm{hNCNC}$ and $\mathrm{PtRu} / \mathrm{hNCNC}$. The top-right insets are HRTEM images and the bottom-right ones of (a, c) are SEM images. (d) Plots of $\mathrm{N}_{2}$ adsorption-desorption isotherms and pore size distribution. (e) XRD patterns. $1 \sim 5$ correspond to Pt/hNCNC, $\mathrm{Pt}_{2} \mathrm{Ru} / \mathrm{hNCNC}, \mathrm{PtRu} / \mathrm{hNCNC}, \mathrm{PtRu}_{2} / \mathrm{hNCNC}$ and Ru/hNCNC (the same below)
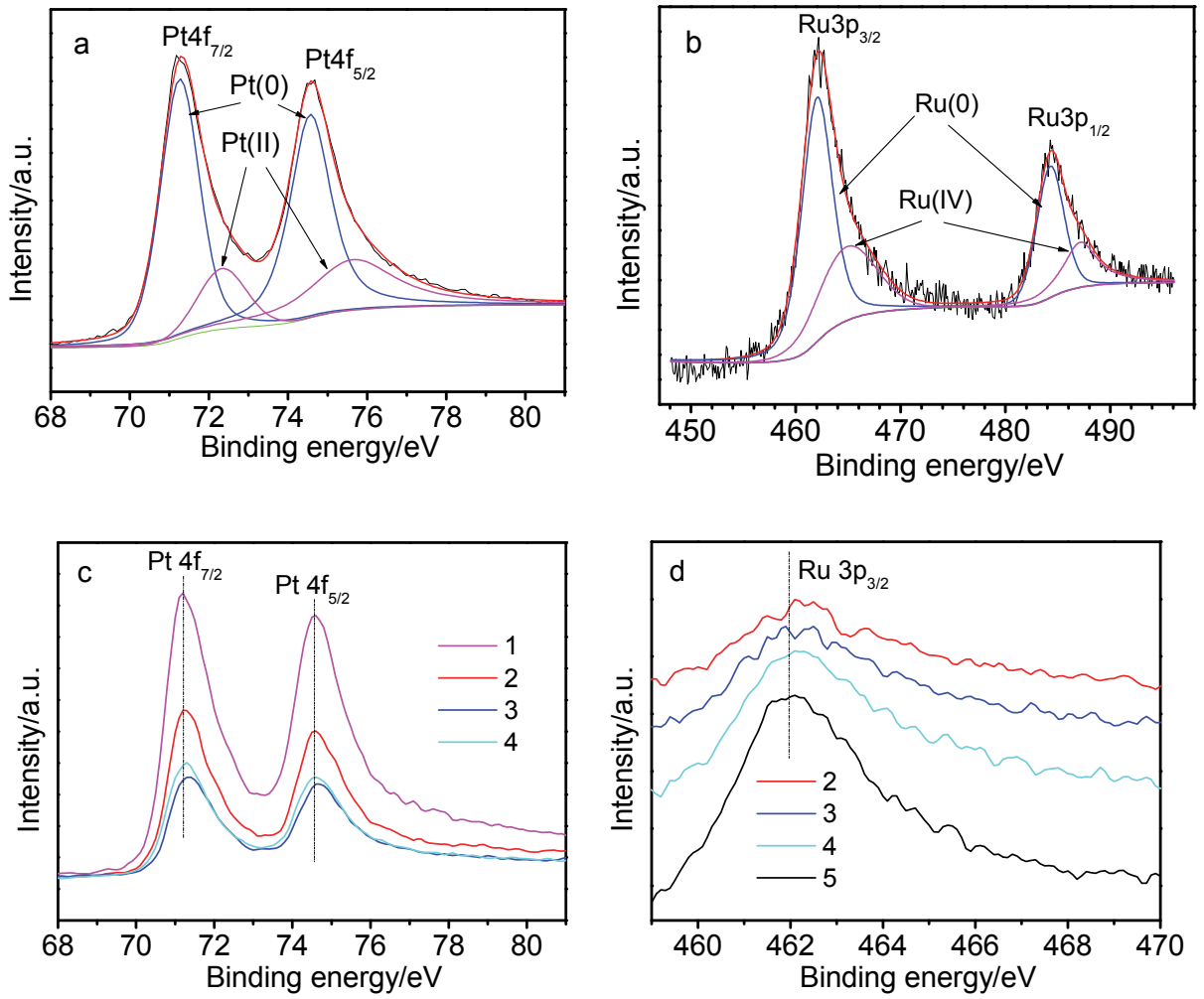

图 $2 \mathrm{Pt}-\mathrm{Ru} / \mathrm{hNCNC}$ 系列样品的 XPS 表征. (a) Pt/hNCNC 的 Pt $4 \mathrm{f}$ 精细谱. (b) Ru/hNCNC 的 Ru 3p 的精细谱. (c, d) Pt-Ru/hNCNC 系列样品的 Pt $4 \mathrm{f}$ 和 Ru 3p 谱

Figure 2 XPS spectra. (a) Pt 4f spectrum of Pt/hNCNC. (b) Ru 3p spectrum of Ru/hNCNC. (c, d) Pt $4 \mathrm{f}$ and Ru 3p spectra for the series of Pt-Ru/hNCNC catalysts 


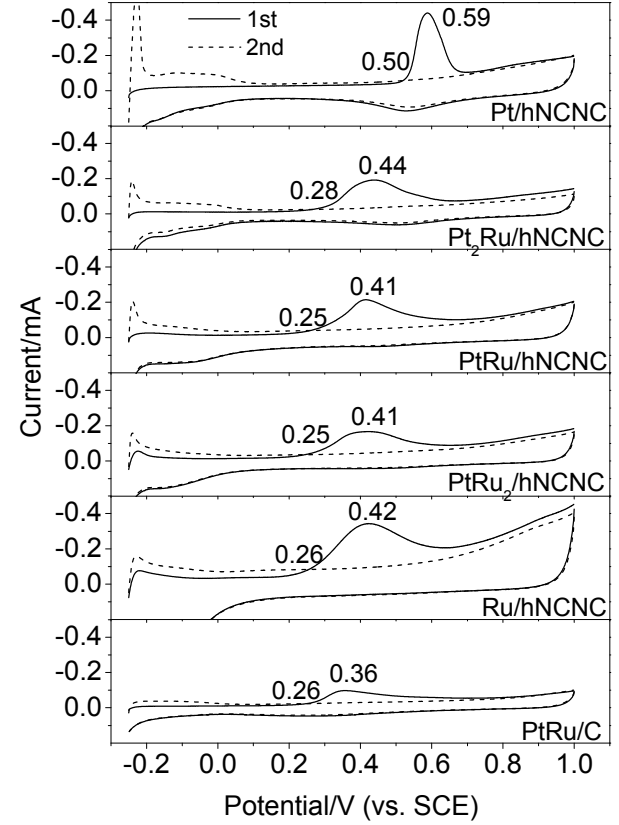

图 $3 \mathrm{CO}-$ 脱除性能. 实线和虚线分别代表第一和第二个循环, 扫速 为 $20 \mathrm{mV} \cdot \mathrm{s}^{-1}, 0.5 \mathrm{~mol} \cdot \mathrm{L}^{-1} \mathrm{H}_{2} \mathrm{SO}_{4}$ 电解液

Figure 3 Cyclic voltammetry (CV) curves of the first (solid line) and second (dashed line) $\mathrm{CO}$ stripping in $0.5 \mathrm{~mol} \cdot \mathrm{L}^{-1} \mathrm{H}_{2} \mathrm{SO}_{4}$ at the scan rate of $20 \mathrm{mV} \cdot \mathrm{s}^{-1}$.

方向偏移了约 $0.2 \mathrm{eV}$, 而 $\mathrm{Ru} 3 \mathrm{p}_{3 / 2}$ 的结合能没有明显的 偏移 (图 2c, 2d). Pt $4 \mathrm{f}$ 的结合能偏移源于 $\mathrm{Pt}$ 和 Ru 合金化 带来的电荷转移引起 $\mathrm{d}$ 价带中心向高结合能位移 ${ }^{[10]}$.

抗 CO 中毒能力是衡量 MOR 催化剂性能的重要指 标. 采用 CO-脱除(CO-stripping)法考察了 Pt-Ru/hNCNC 系列催化剂的电化学活性表面积(ECSA)和去除 CO 的 能力, 结果如图 3 和表 1 所示. 第一圈扫描时, 在 -0.2 $\mathrm{V}$ 附近没有出现氢吸附峰, 在 $0.25 \sim 0.65 \mathrm{~V}$ 区间出现了 $\mathrm{CO}$ 氧化峰, 反映出 $\mathrm{Pt}$ 活性位点被吸附的 $\mathrm{CO}$ 占据. 第 二圈扫描时, 出现了氢吸附峰, 没有出现 $\mathrm{CO}$ 氧化峰, 说明在第一圈扫描时, 被吸附的 CO 已经被完全氧化去 除, 活性位点得以释放(图 3). 从表 1 中可以看出, 随着 $\mathrm{Ru}$ 含量从 0 增加到 $100 \%, \mathrm{ECSA}$ 从 $28.1 \mathrm{~m}^{2} \cdot \mathrm{g}^{-1}$ 增大到 $46.1 \mathrm{~m}^{2} \cdot \mathrm{g}^{-1}$, 高于商业 $20 \% \mathrm{PtRu} / \mathrm{C}$ 的 $21.2 \mathrm{~m}^{2} \cdot \mathrm{g}^{-1}$, 源于

表 1 系列催化剂的 $\mathrm{CO}-$ 脱除和甲醇氧化的电化学数据

Table 1 Electrochemical data from CO-stripping and methanol oxidation for catalysts

\begin{tabular}{cccccccc}
\hline \multirow{2}{*}{ Sample } & \multicolumn{3}{c}{ CO-stripping } & & \multicolumn{2}{c}{ MOR } \\
\cline { 2 - 4 } \cline { 7 - 8 } & $\begin{array}{c}E_{\text {onset }} / \\
\mathrm{V}\end{array}$ & $\begin{array}{c}E_{\text {peak }} / \\
\mathrm{V}\end{array}$ & $\begin{array}{c}\mathrm{ECSA} / \\
\left(\mathrm{m}^{2} \cdot \mathrm{g}^{-1}\right)\end{array}$ & & $\begin{array}{c}I_{\mathrm{f}} / \\
\left(\mathrm{mA}^{\prime} \mathrm{mg}^{-1}\right)\end{array}$ & $I_{\mathrm{f}} / I_{\mathrm{b}}$ \\
\hline $\mathrm{Pt} / \mathrm{hNCNC}$ & 0.50 & 0.59 & 28.1 & & 127.7 & 0.99 \\
$\mathrm{Pt}{ }_{2} \mathrm{Ru} / \mathrm{hNCNC}$ & 0.28 & 0.44 & 29.6 & & 208.2 & 1.58 \\
$\mathrm{PtRu} / \mathrm{hNCNC}$ & 0.25 & 0.41 & 34.4 & & 224.7 & 1.64 \\
$\mathrm{PtRu} / \mathrm{hNCNC}$ & 0.25 & 0.42 & 36.6 & & 163.7 & 2.24 \\
$\mathrm{Ru} / \mathrm{hNCNC}$ & 0.26 & 0.42 & 46.1 & & - & - \\
$\mathrm{PtRu} / \mathrm{C}^{a}$ & 0.26 & 0.36 & 21.2 & & 148.1 & 2.05 \\
\hline
\end{tabular}

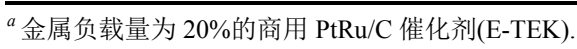

$\mathrm{hNCNC}$ 负载的纳米粒子的均匀分散及小粒径(图 $1 \mathrm{~b}$, $1 \mathrm{c})$. $\mathrm{CO}$ 氧化峰的起始电位 $\left(E_{\text {onset }}\right)$ 和峰电位 $\left(E_{\text {peak }}\right)$ 从 $\mathrm{Pt} / \mathrm{hNCNC}$ 的 0.50 和 $0.59 \mathrm{~V}$ 大幅负向移动到 $\mathrm{Pt}_{2} \mathrm{Ru} / \mathrm{hNCNC}$ 的 0.28 和 $0.44 \mathrm{~V}$; 进一步增加 $\mathrm{Ru}$ 含量, $E_{\text {onset }}$ 和 $E_{\text {peak }}$ 小幅负移到 0.25 和 $0.42 \mathrm{~V}$, 与 $\mathrm{Ru} / \mathrm{hNCNC}$ 的 0.26 和 $0.42 \mathrm{~V}$ 相当. 这说明在 $\mathrm{Pt}$ 催化剂中引入 $\mathrm{Ru}$ 后能够在更低电位下通过氧化有效地去除 $\mathrm{CO}$, 显著提 高催化剂的抗 $\mathrm{CO}$ 中毒能力, 当 $n(\mathrm{Pt}): n(\mathrm{Ru})=1: 1$ 时 表现出了最强的 $\mathrm{CO}$ 去除能力. 抗 $\mathrm{CO}$ 中毒能力的增强 可归因于 Pt-Ru 合金催化剂的双功能机制 ${ }^{[32-34]}$.

采用循环伏安 $(C V)$ 和计时电流 $(I-t)$ 法进一步表征系 列催化剂的 MOR 催化活性和稳定性, 结果如图 4 所示. 在正向扫描过程中, 在 $0.65 \mathrm{~V}$ 左右出现的峰归属于甲醇 氧化 (峰电流密度标记为 $I_{\mathrm{f}}$ ); 在反向扫描过程中, 在 0.45 $\mathrm{V}$ 附近出现的另一个氧化峰归属为中间含碳产物 $(\mathrm{CO}$ 和 类 $\mathrm{CO}$ 物种)的二次氧化 (峰电流密度标记为 $\left.I_{\mathrm{b}}\right)^{[10,35]}$; $\mathrm{Ru} / \mathrm{hNCNC}$ 没有甲醇氧化活性, 与文献结果一致(图 $4 \mathrm{a})^{[36]}$. 由图 $4 \mathrm{a}$ 和表 1 中 $I_{\mathrm{f}}$ 值可知, 催化剂的 MOR 催化 活性顺序为: $\mathrm{PtRu} / \mathrm{hNCNC}>\mathrm{Pt}_{2} \mathrm{Ru} / \mathrm{hNCNC}>$ $\mathrm{PtRu}_{2} / \mathrm{hNCNC}>\mathrm{PtRu} / \mathrm{C}>\mathrm{Pt} / \mathrm{hNCNC}$, 其中 $\mathrm{PtRu} / \mathrm{hNCNC}$ 的 MOR 活性约为商业 $\mathrm{PtRu} / \mathrm{C}$ 的 1.5 倍. $\mathrm{Pt}-\mathrm{Ru} / \mathrm{hNCNC}$ 合金催化剂的优异 MOR 活性可归因于: (1)金属纳米粒
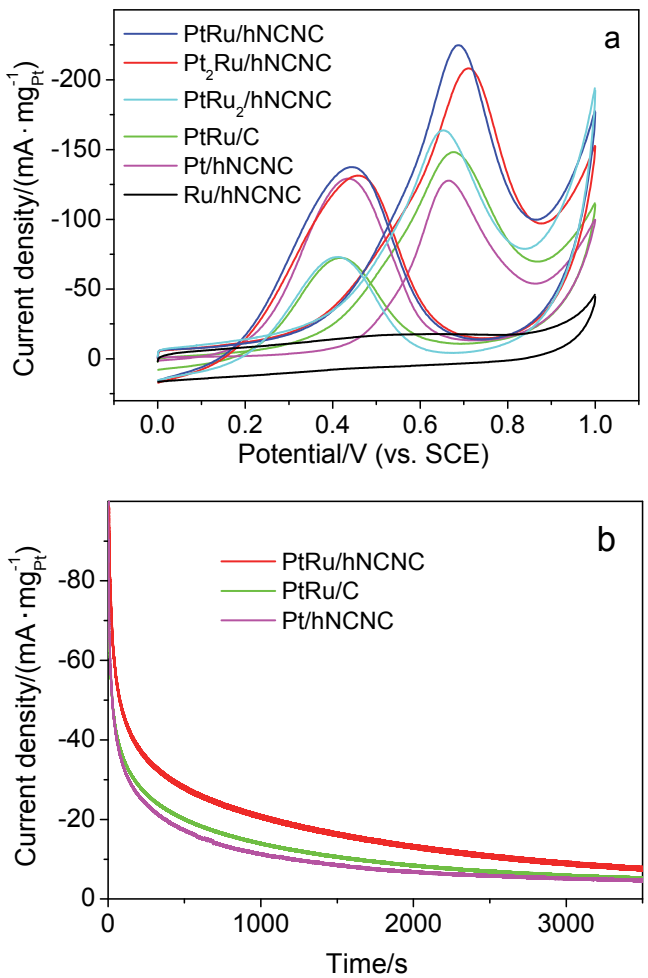

图 $4 \mathrm{MOR}$ 性能. (a) $\mathrm{CV}$ 曲线, 扫速 $50 \mathrm{mV} \cdot \mathrm{s}^{-1}$. (b) 在 $0.6 \mathrm{~V}$ 恒电位下 的 $I-t$ 曲线. 电解液: 氮气饱和的 $1 \mathrm{~mol} \cdot \mathrm{L}^{-1} \mathrm{CH}_{3} \mathrm{OH}+0.5 \mathrm{~mol} \cdot \mathrm{L}^{-1}$ $\mathrm{H}_{2} \mathrm{SO}_{4}$ 溶液

Figure 4 MOR performances. (a) $\mathrm{CV}$ curves at the scan rate of $50 \mathrm{mV}$. $\mathrm{s}^{-1}$. (b) Chronoamperometric (I-t) curves at a constant voltage of $0.6 \mathrm{~V}$. Electrolyte: $\mathrm{N}_{2}$-saturated $1.0 \mathrm{~mol} \cdot \mathrm{L}^{-1}$ methanol and $0.5 \mathrm{~mol} \cdot \mathrm{L}^{-1} \mathrm{H}_{2} \mathrm{SO}_{4}$ solution 
子在 $\mathrm{hNCNC}$ 表面的均匀分散及小粒径, (2)hNCNC 的多 尺度分级孔结构有利于参与反应物质的传输(图 1a～ $1 \mathrm{~d})$. 一般而言, $I_{\mathrm{f}} / I_{\mathrm{b}}$ 比值越高, 表示催化剂抗 $\mathrm{CO}$ 中毒能 力越好 ${ }^{[10,35]}$. 由表 1 的 $I_{\mathrm{f}} / I_{\mathrm{b}}$ 数据可知, 随着 $\mathrm{Ru}$ 含量增加, $I_{\mathrm{f}} / I_{\mathrm{b}}$ 值从 $\mathrm{Pt} / \mathrm{hNCNC}$ 的 0.99 大幅提高到 $\mathrm{PtRu}_{2} / \mathrm{hNCNC}$ 的 2.24 , 该趋势与 $\mathrm{CO}$-脱除的结果相一致(图 3). 图 $4 \mathrm{~b}$ 是 $\mathrm{PtRu} / \mathrm{hNCNC} 、 \mathrm{Pt} / \mathrm{hNCNC}$ 和商业 $\mathrm{PtRu} / \mathrm{C}$ 催化剂在 $0.6 \mathrm{~V}$ 恒电位下的 $I-t$ 曲线. 随着时间延长, 催化剂的电化学活 性均有不同程度的下降, 这可归因于燃料甲醇的消

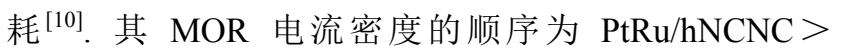
$\mathrm{PtRu} / \mathrm{C}>\mathrm{Pt} / \mathrm{hNCNC}$ (图 4b), 与 MOR 活性一致(图 4a).

\section{3 结论}

利用 $\mathrm{hNCNC}$ 的介观结构特征及掺杂氮原子的针定 作用, 无需任何预处理即可将约 $3 \mathrm{~nm}$ 的 Pt 基纳米粒子 均匀地负载在 $\mathrm{hNCNC}$ 表面, 方法简便. 通过调变 Pt、 $\mathrm{Ru}$ 的物质的量比, 制得了系列 Pt-Ru/hNCNC 合金催化 剂, 展现出优异的 MOR 活性和稳定性及良好的抗 $\mathrm{CO}$ 中毒能力, 显著优于 $\mathrm{Pt} / \mathrm{hNCNC}$ 和商业 $\mathrm{PtRu} / \mathrm{C}$ 等对照组 催化剂. 其中, PtRu/hNCNC 的电化学性能最优, 其 $\mathrm{MOR}$ 活性约为商业 $\mathrm{PtRu} / \mathrm{C}$ 的 1.5 倍. 优异电化学性能 主要源于以下因素的协同作用: (1) Pt-Ru 合金催化剂的 双功能机制; (2) hNCNC 的高比表面积和氮掺杂有利于 金属纳米粒子的分散、针定及粒径的减小; (3) hNCNC 的多尺度分级孔结构有利于物质传输. 本项研究展示了 利用 $\mathrm{hNCNC}$ 独特的介观结构特征开发高性能的负载型 $\mathrm{Pt}$ 基合金 DMFC 催化剂的前景.

\section{4 实验部分}

\section{1 催化剂制备}

采用原位 $\mathrm{MgO}$ 模板法制备 $\mathrm{hNCNC}$ 载体 ${ }^{[27,28]}$. 具体 来说, 将 $4 \mathrm{~g}$ 碱式碳酸镁放入坚式管式炉内, 在 100 $\mathrm{sccm}$ 的氩气保护下, 以 $10{ }^{\circ} \mathrm{C} \cdot \mathrm{min}^{-1}$ 的速率升至 $900{ }^{\circ} \mathrm{C}$, 用平流原以 $60 \mu \mathrm{L} \cdot \mathrm{min}^{-1}$ 的流量通入吡啶 $0.3 \mathrm{~mL}$, 保温 1 $\mathrm{h}$, 自然冷却, 用 $6 \mathrm{~mol} \cdot \mathrm{L}^{-1} \mathrm{HCl}$ 溶液去除 $\mathrm{MgO}$ 模板, 用 去离子水反复洗涤、过滤, $100{ }^{\circ} \mathrm{C}$ 干燥过夜, 即得 $\mathrm{hNCNC}$.

称取 $50 \mathrm{mg}$ 的 $\mathrm{hNCNC}$, 加入 $50 \mathrm{~mL}$ 乙二醇(EG), 超 声 $20 \mathrm{~min}$, 形成均匀悬浊液; 滴入一定量的氯铂酸和三 氯化钉溶液, 磁力搅拌 $60 \mathrm{~min}$; 加入 $2 \mathrm{~mL}$ 的 0.2 $\mathrm{mol} \cdot \mathrm{L}^{-1} \mathrm{NaOH} / \mathrm{EG}$ 溶液, 摚拌 $10 \mathrm{~min}$; 放入微波炉中, 在 $800 \mathrm{~W}$ 微波功率下辐照 $100 \mathrm{~s}$, 冷却至室温; 用乙醇和 去离子水交替洗涤、过滤 3 次, $60{ }^{\circ} \mathrm{C}$ 真空干燥, 即得 $\mathrm{hNCNC}$ 负载的约 $30 \mathrm{wt} \%$ 金属负载量的系列 $\mathrm{Pt}-\mathrm{Ru} /$ $\mathrm{hNCNC}$ 催化剂.

\section{2 结构和成分表征}

采用 TEM(JEM-2100)、SEM(Hitachi S4800)、
XRD(Panalytical X'Pert, Cu 靶, $0.02 \mathrm{~mm} \mathrm{Ni}$ 滤色片)、 XPS(PHI 5000 VersaProbe)、ICP-AES(Hitachi 180-80)表 征催化剂的形貌、结构和成分. 氮气吸附-脱附等温线 (Thermo Fisher Scientific Surfer Gas Adsorption Porosimeter)在液氮温度 $(77 \mathrm{~K})$ 测量, 样品在 $300{ }^{\circ} \mathrm{C}$ 脱气 $6 \mathrm{~h}$. SSA 和孔分布分别用 Brunauer-Emmett-Teller (BET)方 程、Horvath-Kawazoe (HK, $<2 \mathrm{~nm}$ ) 和 Barrett-JoynerHalenda $(\mathrm{BJH},>2 \mathrm{~nm})$ 方法处理吸附支数据得到.

\section{3 催化性能表征}

MOR 性能测试和 CO-脱除实验是在三电极电化学 工作站(CHI760)上完成的. 参比电极和对电极分别是饱 和甘录电极和 $\mathrm{Pt}$ 丝电极. 将玻碳工作电极在抛光布上 用 $50 \mathrm{~nm}$ 的 $\mathrm{Al}_{2} \mathrm{O}_{3}$ 粉抛光, 在无水乙醇中超声 $5 \mathrm{~min}$, 自 然晾干. 将 $2 \mathrm{mg}$ 催化剂分散到 $0.95 \mathrm{~mL}$ 含 $30 \%$ 乙醇(体 积分数)的水溶液中, 另外加入 $50 \mu \mathrm{L}$ 的 Nafion 溶液(5 $\mathrm{wt} \%$ ), 超声 $20 \mathrm{~min}$, 配制成 $2 \mathrm{mg} \cdot \mathrm{mL}^{-1}$ 悬浊液, 用微量 进样器移取 $20 \mu \mathrm{L}$ 上述液体, 滴涂到直径为 $3 \mathrm{~mm}$ 的玻 碳工作电极上, 自然晾干.

\section{References}

[1] Chen, A.; Holt-Hindle, P. Chem. Rev. 2010, 110, 3767.

[2] Kakati, N.; Maiti, J.; Lee, S. H.; Jee, S. H.; Viswanathan, B.; Yoon, Y. S. Chem. Rev. 2014, 114, 12397.

[3] Gasteiger, H. A.; Markovic, N.; Ross, P. N.; Cairns, E. J. J. Phys. Chem. 1994, 98, 617.

[4] Liu, Z. L.; Guo, B.; Hong, L.; Lim, T. H. Electrochem. Commun. 2006, 8,83

[5] Pereira, L. G. S.; dos Santos, F. R.; Pereira, M. E.; Paganin, V. A.; Ticianelli, E. A. Electrochim. Acta 2006, 51, 4061.

[6] Sun, J.; Ma, H.; Jiang, H.; Dang, L.; Lu, Q.; Gao, F. J. Mater. Chem. 2015, 3, 15882 .

[7] Mylswamy, S.; Wang, C. Y.; Liu, R. S.; Lee, J. F.; Tang, M. J.; Lee, J. J.; Weng, B. J. Chem. Phys. Lett. 2005, 412, 444.

[8] Watanabe, M.; Motoo, S. J. Electroanal. Chem. Interfacial Electrochem. 1975, 60, 267.

[9] Yue, B.; Ma, Y. W.; Tao, H. S.; Yu, L. S.; Jian, G. Q.; Wang, X. Z.; Wang, X. S.; Lu, Y. N.; Hu, Z. J. Mater. Chem. 2008, 18, 1747.

[10] Jiang, S.; Zhu, L.; Ma, Y.; Wang, X.; Liu, J.; Zhu, J.; Fan, Y.; Zou, Z.; Hu, Z. J. Power Sources 2010, 195, 7578.

[11] Feng, H.; Ma, J.; Hu, Z. J. Mater. Chem. 2010, 20, 1702.

[12] Joo, S. H.; Kwon, K.; You, D. J.; Pak, C.; Chang, H.; Kim, J. M. Electrochim. Acta 2009, 54, 5746.

[13] Guerrero-Ruiz, A.; Badenes, P.; Rodriguez-Ramos, I. Appl. Catal. A: Gen. 1998, 173, 313.

[14] Kuang, Y.; Cui, Y.; Zhang, Y.; Yu, Y.; Zhang, X.; Chen, J. Chem. Eur. J. 2012, 18, 1522 .

[15] Che, G.; Lakshmi, B. B.; Fisher, E. R.; Martin, C. R. Nature 1998 , 393, 346.

[16] Lin, M. L.; Huang, C. C.; Lo, M. Y.; Mou, C. Y. J. Phys. Chem. C 2008, 112, 867.

[17] Liu, Z.; Su, F.; Zhang, X.; Tay, S. W. ACS Appl. Mater. Interfaces 2011, 3, 3824 .

[18] Yu, J. S.; Kang, S.; Yoon, S. B.; Chai, G. J. Am. Chem. Soc. 2002, 124, 9382.

[19] Li, F.; Chan, K.-Y.; Yung, H.; Yang, C.; Ting, S. W. Phys. Chem. Chem. Phys. 2013, 15, 13570.

[20] Cong, H.-P.; Ren, X.-C.; Yu, S.-H. ChemCatChem 2012, 4, 1555.

[21] Bin, D.; Ren, F.; Wang, H.; Zhang, K.; Yang, B.; Zhai, C.; Zhu, M.; Yang, P.; Du, Y. RSC Adv. 2014, 4, 39612.

[22] La-Torre-Riveros, L.; Guzman-Blas, R.; Méndez-Torres, A. E.; Prelas, M.; Tryk, D. A.; Cabrera, C. R. ACS Appl. Mater. Interfaces 2012, 4, 1134 .

[23] Jiang, S.; Ma, Y.; Jian, G.; Tao, H.; Wang, X.; Fan, Y.; Lu, Y.; Hu, Z.; Chen, Y. Adv. Mater. 2009, 21, 4953. 
[24] Chen, S.; Bi, J.; Zhao, Y.; Yang, L.; Zhang, C.; Ma, Y.; Wu, Q.; Wang, X.; Hu, Z. Adv. Mater. 2012, 24, 5593.

[25] Xie, K.; Qin, X.; Wang, X.; Wang, Y.; Tao, H.; Wu, Q.; Yang, L.; Hu, Z. Adv. Mater. 2012, 24, 347 .

[26] Jiang, Y.; Yang, L.; Sun, T.; Zhao, J.; Lyu, Z.; Zhuo, O.; Wang, X.; Wu, Q.; Ma, J.; Hu, Z. ACS Catal. 2015, 5, 6707.

[27] Lyu, Z.; Xu, D.; Yang, L.; Che, R.; Feng, R.; Zhao, J.; Li, Y.; Wu, Q.; Wang, X.; Hu, Z. Nano Energy 2015, 12, 657.

[28] Zhao, J.; Lai, H.; Lyu, Z.; Jiang, Y.; Xie, K.; Wang, X.; Wu, Q.; Yang, L.; Jin, Z.; Ma, Y.; Liu, J.; Hu, Z. Adv. Mater. 2015, 27, 3541.

[29] Lyu, Z.; Feng, R.; Zhao, J.; Fan, H.; Xu, D.; Wu, Q.; Yang, L.; Chen, Q.; Wang, X.; Hu, Z. Acta Chim. Sinica 2015, 73, 1013. (吕之阳, 冯瑞, 赵进, 范豪, 徐丹, 吴强, 杨立军, 陈强, 王喜章, 胡征, 化学学报, 2015, 73, 1013.)
[30] Feng, R.; Wang, L.; Lyu, Z.; Wu, Q.; Yang, L.; Wang, X.; Hu, Z. Acta Chim. Sinica 2014, 72, 653. (冯瑞, 王立伟, 吕之阳, 吴强, 杨立军, 王喜章, 胡征, 化学学报, 2014, 72, 653.)

[31] Prabhuram, J.; Zhao, T. S.; Liang, Z. X.; Chen, R. Electrochim. Acta 2007, 52, 2649.

[32] Roth, C.; Benker, N.; Theissmann, R.; Nichols, R. J.; Schiffrin, D. J. Langmuir 2008, 24, 2191.

[33] Bock, C.; Paquet, C.; Couillard, M.; Botton, G. A.; MacDougall, B. R. J. Am. Chem. Soc. 2004, 126, 8028 .

[34] Giorgi, L.; Pozio, A.; Bracchini, C.; Giorgi, R.; Turtù, S. J. Appl. Electrochem. 2001, 31, 325.

[35] Wang, Z.-C.; Ma, Z.-M.; Li, H.-L. Appl. Surf. Sci. 2008, 254, 6521.

[36] Liu, S.-H.; Yu, W.-Y.; Chen, C.-H.; Lo, A.-Y.; Hwang, B.-J.; Chien, S.-H.; Liu, S.-B. Chem. Mater. 2008, 20, 1622.

(Cheng, B.) 